\title{
Successful Experimental Quantitative Charge Density Feasibility Study of Grossular Under High Pressure
}

\author{
Roman Gajda ${ }^{a}$, Marcin Stachowicz ${ }^{a}$, Anna Makal ${ }^{a}$, Szymon Sutuła ${ }^{a}$, Pierre Fertey ${ }^{b}$, \\ Krzysztof Woźniak ${ }^{a}$ \\ aDepartment of Chemistry, University of Warsaw, Pasteura 1, 02093 Warszawa, Poland. \\ bSynchrotron SOLEIL, L'Orme des Merisiers Saint-Aubin, BP 4891192 Gif-sur-Yvette Cedex, \\ Paris, France. \\ kwozniak@chem.uw.edu.pl
}

We would like to present results of our high resolution X-ray data collection conducted on the CRISTAL beamline at the SOLEIL synchrotron (Paris, France). We studied single crystal of a natural mineral grossular - $\mathrm{Ca}_{3} \mathrm{Al}_{2}\left(\mathrm{SiO}_{3}\right)_{4}$, which crystallizes in the space group la-3d of the cubic crystal system. We wanted to check if it is possible to determine an experimental electron density of a single crystal under pressure. The beamline parameters such as beam wavelength (0.41 Angstrom) and a special type of Diamond Anvil Cell (DAC) with the opening angle 110 deg. let us collect data with the resolution up to 0.35 Angstrom (with $100 \%$ completeness up to 0.39 Angstrom). We then used CrysAlis(Pro) for data reduction, Olex2 for structure solution and refinement, Jana2006 and XD program packages for multipole refinement, and WinXPro for visualization of different types of maps. We have compared our results with experimental charge densities obtained for grossular at ambient conditions and those obtained for pyrope $\mathrm{Mg}_{3} \mathrm{Al}_{2}\left(\mathrm{SiO}_{3}\right)_{4}$ (isostructural with grossular), measured at low temperature, 30K (Destro at al., 2017) [1]. In the case of our measurements for grossular, the calculated properties of the charge density at the $(3,-1)$ BCPs as well as the net atomic charges are comparable. We think that thanks to the new type of DACs with wider opening angle and access to synchrotron radiations for some types of high symmetry compounds charge density distribution can be determined experimentally. Up to our knowledge this is the first successful determination of quantitative charge distribution in crystal under high pressure. We will present detailed results of our investigations.

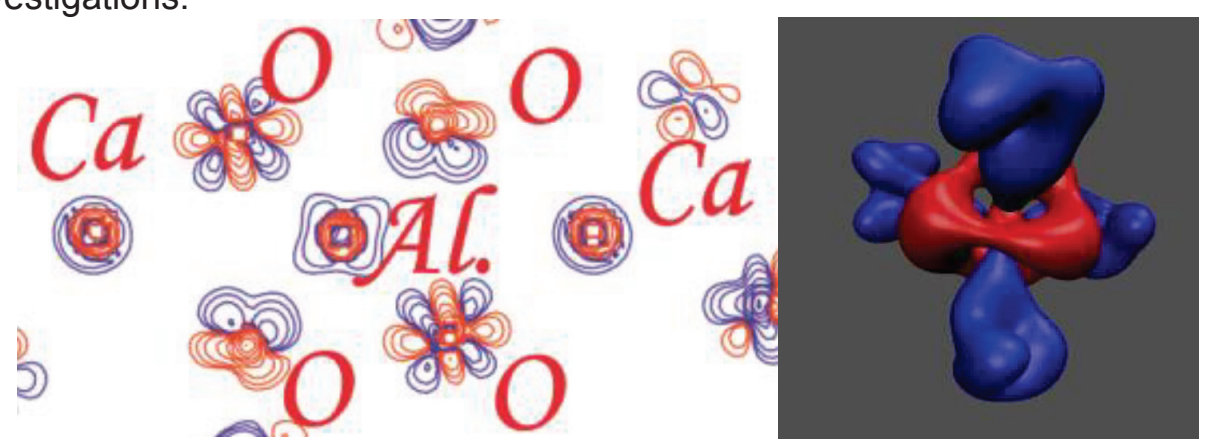

Figure 1. (left) Redistribution of electron density at $\mathrm{O}$ and $\mathrm{Al}$ ions under high pressure, and (right) the $3 \mathrm{D} \mathrm{SiO}_{4}$ deformation electron density maps at ambient conditions - isosurfaces: blue colour $+0.1 \mathrm{e} / \mathrm{A}^{3}$, red colour $-0.1 \mathrm{e} / \mathrm{A}^{3}$.

\section{Reference}

[1] R. Destro, R. Ruffo, P. Roversi, R. Soave, L. Loconte and L. L. Presti, Acta Cryst. B, (2017), 73, 722-736. 